TERRA. Revista de Desarrollo Local

e-ISSN: 2386-9968

Número 9 (2021), 103-121

DOI 10.7203/terra.9.19371

IIDL - Instituto Interuniversitario de Desarrollo Local

\title{
El sistema de la cooperación internacional para el desarrollo: morfología de un paciente crítico
}

\author{
Sascha Quint \\ Docente de la Carrera de Negocios Internacionales en la Facultad de Ciencias \\ Económicas y Administrativas (FACEA) de la Universidad de las Américas (UDLA) - \\ Ecuador \\ sascha.quint@udla.edu.ec
}

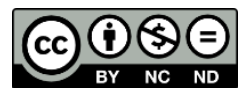

Esta obra se distribuye con la licencia Creative Commons 


\section{SECCIÓN NOTAS Y AVANCES DE INVESTIGACIÓN}

\section{El sistema de la cooperación internacional para el desarrollo: morfología de un paciente crítico}

Resumen: Este artículo ofrece una perspectiva extensa y actual de las críticas que se han realizado a la cadena de valor de la cooperación internacional al desarrollo incluidos objetivos, organización, estructura, acciones y resultados. Para este artículo América Latina fue seleccionada como región de análisis. Se realizó una revisión de literatura de manera integrativa y sistemática. A consecuencia de, se analizaron y sistematizaron las más recientes publicaciones de instituciones de investigación y organismos internacionales involucrados en este tema.

Palabras clave: Cooperación Internacional al Desarrollo, Concepto de Desarrollo, Crisis, América Latina

The system of international cooperation for development: the morphology of a critical patient

Abstract: The article provides a broad and current overview of the criticisms that have been made to the value chain of international development cooperation including objectives, organization, structure, actions and results. For this article Latin America was selected as region under study. For this article, an integrated and systematic review of literature was conducted. As a result, the most recent publications of research institutions and international organizations involved in this topic were analysed and systematized.

Key words: International Development Cooperation, Concept of Development, Crisis, Latin America.

Recibido: 28 de diciembre de 2020

Devuelto para revisión: 22 de abril de 2021

Aceptado: 22 de julio de 2021

Referencia / Citation:

Quint, S. (2021). El sistema de la cooperación internacional para el desarrollo: morfología de un paciente crítico. TERRA. Revista de Desarrollo Local, (9), 103-121. DOI 10.7203/terra.9.19371 


\section{INTRODUCCIÓN}

Después de siete décadas de la práctica de la cooperación internacional al desarrollo en la comunidad internacional (Muñiz de Urquiza (2002) habla de un fenómeno relativamente nuevo en la "sociedad internacional"), y una multitud de literatura producida y publicada sobre los temas de la cooperación internacional, desarrollo internacional y recientemente sobre la eficacia / eficiencia y efectividad de la cooperación internacional, en particular, por parte de instituciones y organismos de desarrollo internacional (Naciones Unidas, Banco Mundial, Unión Europea, Comisión Económica para América Latina y el Caribe, Foro de Cooperación Económica Asia - Pacifico, Organización para la Cooperación y el Desarrollo Económico, Agencia Japonesa de Cooperación Internacional, Agencia Sueca de Cooperación para el Desarrollo Internacional, etc.) (Cabeza-Pulles y Coral-Guerrero, 2016) se ha notado una cierta discrepancia de pareceres sobre la ejecución y el impacto de las actividades cooperativas en la comunidad internacional, y esta diferencia de opiniones se ha convertido en debates altamente politizados entre defensores y oponentes (Ripoll y Ghotme, 2015).

Por un lado, se ve el concepto de la cooperación internacional (al desarrollo) y de la ayuda oficial al desarrollo como un instrumento poderoso que puede de manera considerable facilitar y mejorar la vida de muchas personas al nivel mundial siempre y cuando si existe un entorno adecuado con condiciones adecuadas (por ejemplo, un entorno político estable) que permite un impacto favorable de las actividades cooperativas (Lensink y White, 1999; Unceta y Yoldi, 2000; Alesina, 2002; Sachs, 2004; Islam, 2005; Dollar y Levin, 2006; Collier, 2007; Kasuga, 2007; Thiele et al., 2007; Mekasha y Tarp, 2013; Juselius y Møller, 2014; Arndt et al., 2015; Sow, 2016; Grandas y Prado, 2017; Duarte et al., 2017; Camelo y Mambuscay, 2018; Benito del Pozo y Corcoba, 2019) y por lo menos no argumentan que la ayuda al desarrollo es inefectiva (Easterly et al., 2003).

Por otro lado, la lista de comentarios críticos sobre su razón de ser es muy larga. Partiendo de lo anterior existe una alta cantidad de autores que han mantenido una actitud crítica frente a la cooperación internacional (al desarrollo) sea por una gestión inadecuada de las actividades cooperativas, una baja eficacia, prioridades / objetivos equivocados, el mal uso y / o la mala asignación de recursos escasos, una planificación, monitoreo y evaluación de una calidad moderada, una relación desfavorable entre beneficios y costos de la cooperación internacional hasta inhibiciones burocráticos excesivos o por altas expectativas incumplidas a la cooperación internacional que se ha expresado en una discrepancia existente entre la esperanza de los resultados de la cooperación internacional / asistencia oficial de desarrollo y que se ha logrado hasta ahora en el marco de la cooperación internacional (al desarrollo) en muchas ocasiones. En este sentido autores como Hayek (1953); Friedman (1958); Hayter (1971); Bauer (1972); Mende (1973); Nozick (1974); Moore et al. (1981); Maren (1997); Ridell (2007); Oetzel (2009); Olivié y García (2010); Temple (2010); Alonso y Ocampo (2011); Unceta y Gutiérrez (2012); Grandas (2012); Zamora (2013); Millán et al. (2015); Morgenthau (2015); Narros (2015); Benito del Pozo y Corcoba (2019) se reúnen a la fila de los escépticos criticando a la esencia de la cooperación internacional (al desarrollo) y a la ayuda oficial al desarrollo en general y particularmente en América Latina.

Este artículo de revisión es parte de una investigación en marcha en el marco de una tesis de doctorado del Programa de Doctorado en Desarrollo Local y Cooperación Internacional de la Universidad de Valencia con el título: Análisis de la Incidencia del Biocorredor Cayambe - Coca del Programa de Pequeñas Donaciones (PPD) en el 
desarrollo económico local de las poblaciones en las parroquias Olmedo (Pesillo), Cangahua, Oyacachi y Angochagua en el plazo de 2011-2016.

El artículo radica en un acercamiento a las críticas específicas actuales a lo largo de toda la cadena de creación de valor añadido que se han acumulado y expresado en los últimos años en el ámbito de la cooperación internacional al desarrollo y la ayuda oficial al desarrollo de varios autores de academia e instituciones internacionales con un mayor enfoque en América Latina.

\section{METODOLOGÍA}

Para este artículo se realizó un estudio detallado, selectivo y crítico de la literatura pertinente utilizando los descriptores en Español: "Cooperación Internacional al Desarrollo", "Desarrollo", "Cooperación Internacional y sus críticas" y "Cooperación Internacional al Desarrollo en América Latina" en idioma anglosajón; "International Development Cooperation" "International Aid", "Económic Development", "Económic Development and International Aid", "International Development Cooperation and Criticism" and "International Development Cooperation in Latin America" también en el idioma de Alemán: "Internationale Entwicklungszusammenarbeit", "Entwicklungshilfe", "Kritiken an der internationalen Entwicklungszusammenarbeit", "Kritiken an der Entwicklungshilfe" e "Internationale Entwicklungszusammenarbeit in Lateinamerika".

Se realizó esta búsqueda mediante las bases de datos Web of Science. A parte, la búsqueda bibliográfica se concentró en la base multidisciplinar de datos de Scopus, Se extendió la búsqueda bibliográfica tomando en cuenta otros tipos de documentos como libros, guías y manuales (Handbooks), tesis y disertaciones y, por último, páginas web de instituciones internacionales involucradas en el tema de la cooperación internacional al desarrollo.

\section{EL SISTEMA DE LA COOPERACIÓN INTERNACIONAL AL DESARROLLO: UN PACIENTE CRITICO}

Si fuera la cooperación internacional al desarrollo un ser humano, el mismo estaría muy enfermo a pesar de encontrarse en el otoño de la vida con alrededor de 75 años, sufriendo de un cuadro clínico complejo y crónico. El diagnóstico sería un "síndrome de fatiga crónica". Las personas afectadas muestran un cansancio intenso y continuo que provoca perjuicios a la calidad de vida de los enfermos. Si el sistema de la cooperación internacional representaría la comunidad mundial hablaríamos en este contexto de una pandemia de agotamiento extremo (una enfermedad que se extiende a nivel mundial).

\subsection{Agotamiento, Desequilibrio y Olvido}

La situación del paciente "Sistema de Cooperación Internacional al Desarrollo" es grave y se habla muy a menudo de una "fatiga - un cansancio permanente de los estados donantes" (Lucas y Franco, 2017, pp. 10-11 y 19-20), de un "cansancio y quebranto de la voluntad política de algunos estados industrializados, entre ellos los más ricos" (Pearson, 1970, p. 8), de la "impotencia en los países donantes" (Muñoz Ocaña, 2013, p. 39) o de 
la "frustración de la cooperación", un desgaste por parte de los cooperantes (Posadas Mijangos, 2013, p. 100) que tiene que ver con la reducción gradual de los flujos de la ayuda oficial al desarrollo en la comunidad internacional, por los pocos avances e impacto positivo que han generado las transferencias de la ayuda oficial al desarrollo en los países socios. Muñoz (2013, p. 39) destaca en este sentido que esta disminución de los flujos de la ayuda oficial al desarrollo sostiene el peligro que los estados donantes tienen la "excusa perfecta (...) para dejar de ocuparse de los problemas de las poblaciones empobrecidas de los países socios, dejándolos abandonados a su suerte".

La mala condición en que se encuentra el "Sistema de Cooperación Internacional al Desarrollo" ha inspirado a una serie de autores de describir el mismo de una manera más o menos metafórica como los siguientes ejemplos en la literatura relevante indican; se describe el sistema como un conjunto de relaciones y estructuras débiles, desequilibradas y no confiables para todas partes (Alonso, 2001; Sogge, 2009; Domínguez, 2017), es un sistema sin memoria que es "...tan alto el edificio de la cooperación que a menudo quienes construyen sus últimos pisos ya han olvidado sobre qué bases están parados" (Sábato et al., 1981, p. 18; Domínguez, 2017, p. 111).

\subsection{Frankenstein, Zombis y el Lado Oscuro}

El autor Lo Brutto (2017, p. 71) habla de un nuevo "Frankenstein" de la ayuda que se ha generado y hace referencia a la tendencia en el sistema de cooperación internacional al desarrollo (SCID) de querer "....cambiar radicalmente lo que hasta ahora ha sido entendido como (SCID)" para cumplir con los 17 Objetivos de Desarrollo Sostenible de la Agenda 2030 sobre el Desarrollo Sostenible, criticando la ausencia de una reflexión más profunda sobre el pasado de la ayuda oficial para el desarrollo y su papel inicial en la cadena de valor del sistema de cooperación internacional. En cambio, se ha producido una estructura artificial de enorme estatura de la cooperación internacional para el desarrollo que está marcada de una disformidad física y que su vida es resultado de un experimento. Frankenstein manda saludos, un ser creado artificialmente de partes diferentes de cadáveres que nos hace recordar que el sistema de cooperación internacional es un sistema "Zombi”, muerto, pero a su vez todavía vivo, un “...viejo sistema que se resiste a morir y el nuevo que no acaba de nacer" (Lo Brutto, 2017, p. 71). Es un sistema perverso con respeto a los incentivos que existen en el marco de la ayuda oficial al desarrollo para todos los actores involucrados (Easterly, 2006). Lucatello (2017) encuentra en el sistema de la cooperación internacional "lados oscuros".

\subsection{Fracaso, Crisis y Dilema}

Otros autores se pierden en descripciones más generales sobre el estado actual del sistema de la cooperación internacional concluyendo que la cooperación internacional al desarrollo y la ayuda oficial al desarrollo y sus formas antiguas han sido un fracaso (Oroval, 2008; Domínguez et al., 2009; Vassalli y Rodriguez, 2017), el sistema de la cooperación internacional se encuentra en una "vigente crisis" (Monje, 2018, pp. 95-114), en una "profunda crisis" (Cuñego y Ruíz 2015, pp. 38-39), en "tiempos de crisis" (Encinas, 2013, p.1) o en un dilema como opinan Unceta y Yoldi (2000, pp. 90-91) y Jiménez (2018, p. 224), por qué el “...sistema de cooperación internacional al desarrollo 
es epistemológica y operativamente ajeno a la complejidad reinante en su mundo de actuación".

Por esta razón para Llistar (2009) la cooperación internacional no tiene mucho sentido en su actual forma y estructura como instrumento para disminuir la pobreza, mantener ecosistemas o proteger derechos humanos al nivel mundial. Según el mismo autor la cooperación internacional ha provocado al contrario un aumento de la pobreza, la destrucción de ecosistemas y ha dado espacio para la violación de derechos humanos, ha forzado el comercio injusto, no ha disminuido la guerra o ventas de armas y ha erosionado la soberanía alimentaria en el mundo con flujos transnacionales monetarios y físicos crecientes.

Llistar (2009, p. 55) usa y propone por esta razón el prefijo "Anti” antes del morfema "Cooperación" y desarrolló el término "Anticooperación." Es una expresión introducida por el mismo autor que pone énfasis en las interferencias negativas que ha causado la cooperación internacional (Anticooperación) desde el hemisferio del Norte cuyos efectos sean directa o indirectamente dañosos para el Sur. En otras palabras, es actuar para un fin propio y conveniente con afectación negativa a un tercero. Llistar menciona en este sentido algunos ejemplos que sostienen su concepto de la "Anticooperación" desde el Norte hacia el Sur como los “... apoyos estatales a la internacionalización de la empresa que pueden generar deuda externa ilegítima, suelen estar fuera de control; la presión que generan los nuevos objetivos en el uso de agrocombustibles de EEUU y la Unión Europea sobre los campesinos que disponen de tierras fértiles en zonas tropicales; el apoyo a un régimen autoritario por motivos geoestratégicos con la venta de armas y la consecuente represión — muerte incluida — de decenas de miles de civiles” (Llistar, 2009, p. 59).

\subsection{Mito, Mentira y Muerte}

Otros autores van un paso más allá destacando que la buena y potente ayuda que alivia la pobreza sistémica al nivel mundial y de que en realidad lo ha hecho es un gran "mito", la ayuda internacional es un concepto muerto ("Dead Aid") (Moyo, 2011) y Durán Blanco (2008) consta finalmente que la cooperación internacional es una "gran mentira".

El "Sistema de Cooperación Internacional al Desarrollo" es para una multitud de sus críticos un paciente que sufre de apatía, se trata de un paciente olvidadizo, desorientado con agonía con una estatura desproporcionada que se encuentra en una posible crisis de edad. El paciente ha logrado una cierta popularidad mundialmente moviéndose entre una notable jerarquización Norte / Sur acompañada de una marcada visión neoliberal que se caracteriza por "... acciones de cooperación centradas en lograr una eficacia de la ayuda dentro de los mismos esquemas neoliberales..." (Lo Brutto, 2017, pp. 66-67). Sin embargo, es un paciente que se encuentra en la búsqueda de identidad, incapaz de cumplir de “...generar el tipo de eficacia..." que el mismo plantea en sus “... programas y proyectos que se han implementado a lo largo y ancho de los países en desarrollo y subdesarrollados." Por esta razón Lo Brutto (2017, pp. 66-67) le certifica a este paciente bajo las condiciones en que se encuentra actualmente sombrías perspectivas de futuro y las posibilidades de recuperación del paciente son moderadas. El paciente con una cara siniestra y sombría ha perdido del sentido de la realidad. Sin embargo, este paciente sigue vivo, él no tiene muerte cerebral. Se tiene que ser paciente con él. La esperanza de mejorar muere al final. 


\section{LA COOPERACIÓN INTERNACIONAL AL DESARROLLO: VOCES CRÍTICAS DESDE AMÉRICA LATINA}

En América Latina la intervención de la cooperación internacional al desarrollo ha sido parte de la realidad regional desde los años 70 . Casi 50 años de inserción han hecho surgir muchas esperanzas de solucionar problemáticas locales, regionales y nacionales, por otro lado, han provocado una seria de decepciones especialmente en los países en vías de desarrollo. En otras palabras, el albazo (un género musical de origen ecuatoriano con un ritmo alegre) se enfrenta con toda su fuerza a un pasillo ecuatoriano (un género musical lento, melancólico y triste) en al ámbito de la cooperación internacional al desarrollo. Sin embargo, no se oculta la crisis en la que se encuentra la cooperación internacional al desarrollo en este rincón del mundo.

\subsection{Rutina, Comodidad e Inercia}

Autores como Acosta (2010) critican particularmente para América Latina una obvia rutina que han mostrado las actividades de la cooperación internacional, que se manifiesta en el comienzo de la entera cadena de valor del sistema de la cooperación internacional, en la definición de sus objetivos, y se ha culminada en una comodidad y así en una inercia del sistema de la cooperación internacional que ha causado en muchas ocasiones la necesidad de un reajuste de varios programas que no están bien alineados a las verdaderas necesidades específicas de las personas que deberían ser beneficiadas de la asistencia al desarrollo. Si se toma en cuenta que la cooperación internacional es una herramienta política (en muchas ocasiones política económica) de los países con frecuencia del mundo occidental y este instrumento está adaptado al corriente término del "desarrollo" de estos países, autores como Acosta (2010) critican la asistencia al desarrollo de varios países latinoamericanos fijándose en el hecho que la cooperación internacional es un catalizador de la implantación de un concepto de desarrollo con una alta influencia neoliberal que incluye el reduccionismo del concepto de desarrollo económico al concepto de crecimiento económico expresado en un paquete de actividades mínimo como la transferencia de recursos, materiales y conocimientos desde el Norte hacia el Sur.

Gudynas y Acosta (2011) se alejan de esta reducción proponiendo la necesidad que es ineludible de concentrase en una alternativa al concepto de desarrollo y no a conceptos de desarrollos alternativos que proponen muchos programas en el ámbito de la cooperación internacional en América Latina (Acosta, 2010, Gudynas y Acosta, 2011, Gudynas, 2011).

\subsection{Objetivos Distorsionados, Instrumentos Inadecuados y Estrategias Obsoletas}

Oroval (2008) cuestiona a la cooperación internacional sus objetivos distorsionados especialmente que todavía después de siete décadas de la existencia de esa forma de ayuda todavía se enfoca especialmente en el desarrollo económico y en "...su instrumento básico, la transferencia de recursos", y deja por un lado la “...creciente desigualdad en la distribución de la riqueza, del bienestar y de las oportunidades". Para el mismo autor la cooperación internacional al desarrollo es básicamente un instrumento que facilita el 
proceso de la internacionalización de los intereses económicos y políticos de las empresas e instituciones occidentales que oficialmente ofrecen ayuda al Sur en forma transferencias de recursos. Oroval (2008) concluye que la cooperación internacional ofrecida por el Norte "...responde fundamentalmente a los intereses del estado y de las empresas con capital..." del Norte y que estos intereses están por “...encima de las lógicas filantrópicas o de justicia social". A través de objetivos fallidos definidos por parte del aparato de la cooperación internacional se debe repensar el papel de la cooperación internacional en América Latina. Oroval (2008) detalla y demanda una "repolitización de la cooperación al desarrollo" que implica la necesidad que deberíamos entender la cooperación internacional al desarrollo "...como un elemento de cambio, como un instrumento para luchar contra las injusticias..." que requiere "otros instrumentos y nuevas estrategias" (Oroval, 2008, pp. 1-3).

\subsection{Asistencialismo, Formalismo y Generalismo}

Torres consta que la cooperación internacional no es parte de la solución, sino parte del problema, en el sistema internacional criticando el hecho que especialmente en el sector educativo en América Latina la intervención de la cooperación internacional “...ha sido tradicionalmente invisible y lejana para la gran mayoría de las personas, e incluso para los investigadores y especialistas de la educación" (Torres, 2000, pp. 3-4).

Los autores Domínguez et al. (2009) y Gallicchio (2004) destacan la falta de la efectividad de la cooperación internacional por su carácter "asistencialista, formalista y generalista" en Latinoamérica que no le ha permitido de disminuir problemas existentes de las personas por ejemplo la reducción de la pobreza, y de esa manera no ha apoyado en bajar la delincuencia, el narcotráfico y la emigración en "Estados frágiles" en la zona latinoamericana. Gallicchio (2004) adiciona que los programas de la cooperación internacional han fallado en los países latinoamericanos. "No hay evidencia concluyente de que hayan promovido sostenidamente desarrollo económico y social en los países de América Latina. La pobreza no ha bajado y la exclusión es creciente." (Gallicchio, 2004, p. 71) Por esta razón Domínguez et al. (2009, pp. 63-66) proponen una nueva forma de cooperación internacional en América Latina, una “...cooperación más proactiva y de intervención directa-pactada, donde se generen y gestionen nuevos servicios y se ejecuten, sin distorsiones, la mayor parte de las inversiones”. Esto implica negociaciones francas, duras y condicionadas con los países acostumbrados a los recursos de la cooperación, mediante fórmulas de cooperación triangular y, a su vez, una gran alianza internacional entre gobiernos donantes, organismos multilaterales, empresas multinacionales socialmente responsables y organizaciones no gubernamentales en vías de empresarización (Domínguez et al., 2009).

Lallande (2005) detalla que "...la ayuda falla tras no apoyar decididamente a los pobres. Esto es así porque la ayuda se otorga básicamente por la vía gubernamental, y debido a que los gobiernos representan intereses particulares, estos recursos, el lugar de apoyar a los sectores más desprotegidos, han beneficiado en mayor proporción a las élites de los países receptores" (p. 8). Gallicchio (2004, p. 72) adiciona que el apoyo de la cooperación internacional sería mucho más impactante si los actores involucrados se fijarían más en la transferencia de capacidades y no sólo en recursos. 


\subsection{Falsas Prioridades, Necesidades Desconocidas y una Débil Coordinación}

Según la Comisión Económica para América Latina y el Caribe (CEPAL) existe la necesidad de un cambio en la política y en las prioridades con respeto a la asignación de recursos por parte de la asistencia oficial de desarrollo. La misma organización cuestiona la lógica de reducir y/o quitar recursos para países latinoamericanos que están oficialmente clasificados países de renta media para garantizar en el mismo tiempo un flujo de recursos a los países que son declarados países de renta baja. Esta política perjudica un avance en el desarrollo en los países con economías emergentes provocando una nueva restricción o escasez interna de recursos en estos países que puede causar por su parte retrocesos en el entorno social en la región latinoamericana y frenar los avances con respeto en cumplir con los Objetivos de Desarrollo de Milenio.

La política de la asignación de recursos de la asistencia oficial de desarrollo no debería perseguir de manera ciega a algunos índices económicos sino apuntar de manera adecuada a las necesidades verdaderas de los países o en palabras de la Comisión Económica para América Latina y el Caribe (CEPAL) “...las prioridades de asignación de asistencia deberían fijarse sobre la base de un conjunto de indicadores más amplio que refleje las particularidades concretas de los países y permita detectar dónde están las principales necesidades en cada caso particular" (CEPAL, 2010, p. 24). Esto es posible a través de una comunicación transparente y honesta entre los países receptores y donantes. El intercambio de información entre países donantes y receptores debería "...determinar cuáles son las áreas prioritarias y detectar las necesidades de financiamiento no cubiertas en cada una de esas áreas. Esto no solo serviría para identificar con exactitud las demandas principales de cada país, sino también para solucionar ciertas fallas de coordinación que surgen entre receptores y donantes y por las que muchas veces ciertas áreas con menores brechas de financiamiento terminan recibiendo mayores volúmenes de asistencia en términos relativos que otras con mayores brechas dentro de un mismo país" (CEPAL, 2010, p. 24).

Para los países desarrollados o en vías de desarrollo, países receptores y donantes la CEPAL propone en este sentido nuevas formas innovadoras de financiamiento “...que se sumen a las que actualmente se utilizan y puedan aportar recursos frescos ante una coyuntura de escasez general de financiamiento..." (CEPAL, 2010, p. 25). Ante esto CEPAL propone otras formas de la cooperación internacional que pueden ser una alternativa válida a la que de la asistencia oficial de desarrollo; en este caso las formas de la cooperación Sur-Sur (un tipo de colaboración entre diversos países en desarrollo en el marco político, económico, social, tecnológico, cultural y ambiental que se puede presentar de manera bilateral, regional, subregional e interregional. En esta forma de asociación de cooperación los países involucrados comparten conocimientos, habilidades, experticia y recursos para alcanzar sus metas de desarrollo a través de esfuerzos concertados) y la Cooperación Triangular (un concepto que abarca la participación de mínimo dos países en vías de desarrollo que puede contar con una cierta contribución de una tercera parte, por lo general un país desarrollado, una economía emergente y/o una organización multilateral con el objetivo principal de compartir soluciones para el desarrollo, también conocimientos, capacidades, experiencias y buenas prácticas, tecnología y otros recursos). 


\subsection{Neo - Colonialismo, Eurocentrismo e Indicadores Paralelizados}

Para Pearson (1970) y Restrepo (2012) la cooperación internacional al desarrollo ha sido un instrumento neo - colonizador y eurocentrista en la región latinoamericana. Restrepo explica que diversos actores del norte “...se han apoderado de las problemáticas sociales de nuestras naciones, buscando dar les una mirada ajena a la realidad que las rodea" ( $p$. 294). La misma autora no solo critica que las políticas de la cooperación internacional al desarrollo introducidas al Sur son de entidades que tienen su origen en el hemisferio del norte, sino también reclama el asunto que estas políticas “...no han logrado cambios trascendentales en el panorama de derechos humanos de la región y se han atomizado en la distribución inequitativa de recursos a regiones, que tal vez, tienen más importancia para sus países desarrollados" (Restrepo, 2012, p. 294).

Restrepo (2012) hace responsable el comportamiento incorrecto de la cooperación internacional al desarrollo por los indicadores de desarrollo paralelizados y estáticos en Latinoamérica y consta que "...los donantes y operadores de CID se olvidaron de la importancia de los instrumentos internacionales de derechos humanos como base fundamental y condición sine qua non de sus proyectos. El enfoque de derechos humanos entonces no deja de ser más que el rediseño de la ya justificada CID, buscándole salidas a un modelo que, evidentemente, ya fracaso" (p. 294).

\subsection{Ignorancia, Asimetría de Intereses e Interdependencia}

El enfoque positivista y legitimadora de los Estudios del Desarrollo del sistema de la cooperación internacional al desarrollo causa una cierta ignorancia al frente de "...las experiencias de negociación y demandas económicas" de los actores receptores de la ayuda oficial al desarrollo. En este sentido se encuentran enfrentados “...las experiencias de negociación y demandas económicas de los receptores de ayuda (financiación pública neta para la industrialización y la convergencia), y la oferta 'mucho más política' de los donantes" (Domínguez, 2017, p. 111).

La zona geográfica latinoamericana parece un ejemplo adecuado para Domínguez que corrobora lo que se ha mencionado con respeto a las experiencias de negociación y demandas económicas, una región que ha sido considerada por parte de los estados donantes (especialmente los EEUU) como aliada fiable y que ha sido caracterizada como zona relativamente más desarrollada según los Estudios del Desarrollo que entonces ha perdido “...peso como región receptora” (Domínguez, 2017, p. 111).

Domínguez encuentra en el sistema de la cooperación internacional al desarrollo una asimetría y desarmonía de intereses entre Estados y actores de la sociedad civil global que causa un alto grado de interdependencia compleja entre los actores involucrados. En este sentido el mismo autor destaca que se trata sobre una "interdependencia entre desiguales" y apunta que "todos [los países] somos interdependientes, pero unos son menos interdependientes que otros [...] Hay interdependencia positiva e interdependencia negativa. Dentro de ese orden internacional asimétrico se reconocen intereses divergentes negociados en torno a determinadas normas e instituciones [...], que unos países defenderán si les benefician y otros querrán cambiar si les perjudican. Por tanto, el orden internacional supone ciertas expectativas de estabilidad y consenso, pero no la ausencia de conflictos y contestación [...]" (Domínguez, 2017, p. 112). 


\subsection{Presencia excesiva de agencias de la Cooperación Internacional al Desarrollo, Discrepancia entre las prácticas de la cooperación y la aplicación del desarrollo y una Gestión Inadecuada}

Lucatello (2017) nota la alta cantidad y su influencia considerable de los agentes de la cooperación internacional al desarrollo especialmente en forma de las ONG en América Latina. Esta masiva presencia “...ha añadido un componente de variabilidad social de gran relevancia en el análisis del contexto político" en la zona geográfica latinoamericana. Las acciones y actividades de las ONG “...han sido particularmente afectadas por los cambios y las transformaciones de los últimos dos o tres decenios y su rol a favor de una mejora de las condiciones de la sociedad..." (p. 166) En este sentido el mismo autor critica que las ONG y sus intervenciones no han cumplido siempre con su papel de contribuir al logro de una sociedad mejor en América Latina. Al contrario, el mismo autor habla de una "...cooperación tóxica [...] esbozando la compleja relación entre las organizaciones de la sociedad civil (ONG) en América Latina y su simbiosis con el desarrollo y la cooperación" (Lucatello, 2017, p. 186).

El "boom" de las materias primas y de los commodities al nivel mundial y relacionado con esto una fuerte subida de sus precios ha aún más envenenado la relación entre la cooperación y el desarrollo. Lucatello (2017, p. 190) detecta entonces “...espirales peligrosas entre las prácticas de la cooperación y la aplicación del desarrollo, incluyendo las llamadas reformas estructurales en países como México y Brasil". Esta relación desfavorable entre la cooperación internacional al desarrollo y el desarrollo en Latinoamérica ha causado un "... escepticismo creciente y una resistencia cada vez más marcada por parte de las poblaciones de la región" que ha creado a su vez un ambiente de "...desafíos para ejercer un mejor y mayor control de la agenda global de la eficacia de la cooperación y de sus actores implementadores".

Lucatello, (2017) y Monje (2018) concentran en este contexto sus críticas sobre la cooperación internacional en el modelo de gestión implementado en muchos programas de la cooperación internacional en la región latinoamericana. Monje (2018, p. 95) habla de un "agotamiento del modelo de gestión implementado" y nota que por las últimas décadas gran parte de los proyectos de la cooperación internacional al desarrollo ejecutados "...no han sido capaces de contribuir de manera significativa y sostenible con la mejora de las condiciones de la vida de las poblaciones más pobres y excluidas de los países en vías de desarrollo." A causa de este hecho existen dudas considerables con respecto "... a la forma como se ha gestionado la cooperación internacional y, sobre todo, al modelo de desarrollo promovido" (Monje, 2018, p. 95).

Monje (2018) destaca en este sentido la importancia de la participación empoderada de la población destinataria para garantizar mayores éxitos de los proyectos de cooperación internacional al desarrollo y critica en el mismo tiempo la existente ausencia de proyectos ejecutados más integradoras con un enfoque más participativo en América Latina. Según el mismo autor "...no existe un modelo realmente "horizontal" de promoción de desarrollo que facilite el éxito de los proyectos ejecutados a través de dicho tipo de participación." Este autor concluye entonces que “...existen fuertes indicios para suponer que en muchos proyectos de cooperación internacional al desarrollo implementados en la región latinoamericana se están subordinando los intereses de la población objetivo a los intereses particulares de las agencias de cooperación internacional y de sus respectivos gobiernos" (Monje, 2018, pp. 95 y 114). 


\subsection{Modesto Impacto, Baja Efectividad y Lentos Avances}

Larrera hace énfasis en el impacto de la cooperación internacional a los sectores sociales y económicos en el Ecuador y admita por un lado una importancia del apoyo internacional que se refleja en una tendencia ascendente del 3,4\% al 4,5\% del PIB entre 1990 y 2001 en este país. Se puede confirmar que la cooperación internacional en Ecuador ha sido una parte estratégica del Gobierno con respeto a varias inversiones nacionales realizadas en las áreas de infraestructura, desarrollo social y modernización del Estado Ecuatoriano. Por otro lado, Larrera (2003) cuestiona la baja efectividad de las acciones de la cooperación internacional mencionando "...los limitados efectos sociales de las estrategias de desarrollo adoptadas por el país y la región en las últimas décadas, sino también a temas más específicos como la baja efectividad o reducida focalización de las inversiones para el desarrollo social y la escasa correspondencia entre la composición sectorial de los proyectos financiados y las prioridades del desarrollo" (p. 2).

\section{CONCLUSIONES}

Sin duda, en los últimos años se ha demostrado una creciente fragmentación de la cooperación internacional al desarrollo que ha intensificada la necesidad de una permanente retroalimentación y reflexión sobre su espectro de prestaciones y potencias incluidos sus objetivos, gestión y resultados en el ámbito de desarrollo. Esta inquietud mostrada no ha aparecida de un día a otro, sino ha tenido su cierta trayectoria en forma de una seria de consensos, cumbres, conferencias y foros diferentes organizados por parte de los protagonistas del sistema de la cooperación internacional al desarrollo.

Las discusiones que se llevaron a cabo en estos eventos o denominados hitos muestran que los frentes entre los defensores y opositores sobre el rumbo adecuado de la cooperación internacional al desarrollo se han endurecido. La balanza entre concurrencia y critica con respeto al trabajo de la cooperación internacional al nivel mundial parece relativamente equilibrada. Este debate se intensifica por la existencia de una alta cantidad de críticas de cualquier índole al modelo tradicional de la cooperación internacional al desarrollo, lo cual no es muy sorprendente, por qué se trata de un constructo que ha sido en realidad un término corriente en el transcurso de las últimas décadas, un término que se ha cambiado con frecuencia en el pasado a causa de una salida y entrada intensa de variables que han influido en la definición de la cooperación internacional al desarrollo, adaptándose con éxito mixto al constructo de "Desarrollo", que ha resultado también un carácter cambiante hasta ahora.

El sistema de la cooperación internacional al desarrollo no es perfecto y la interacción de las entidades particulares en este sistema no funcionan a la más entera satisfacción. En otras palabras, la anatomía de la crisis del paciente de "la cooperación al desarrollo" reveló síntomas fuertes que se muestran en una multitud de actores con intereses particulares que definan objetivos y después tratan de cumplir con los mismos. Al final se intenta de medir los resultados de su intervención cada uno a su manera. La debilidad del sistema se muestra además en la competencia entre los estados donantes "... en la financiación de proyectos y programas, empleando gestores y consultores con las mismas habilidades y conocimientos, creando estructuras y procesos paralelos para crear, 
gestionar y evaluar sus intervenciones" (Aristizábal, 2010, p. 39). También en la exigencia de los estados donantes "... a los receptores que cumplan con diferentes regulaciones y procedimientos, mientras que los receptores tienen que destinar escasos recursos humanos y tiempo para interactuar con todos los donantes y sus proyectos" (Aristizábal, 2010, p. 39), y en una alta volatilidad de la ayuda oficial al desarrollo y de su destino y pocos incentivos para los países donantes y receptores de cambiar el estatus quo del sistema de la cooperación internacional al desarrollo (Aristizábal, 2010). Finalmente cabe mencionar que todas estas irregularidades o síntomas en el sistema de la cooperación internacional al desarrollo no se han eliminado o están en un proceso de mejora muy lentamente.

Se ha considerado en este artículo el concepto de la cadena de valor. Este enfoque analítico no es nuevo en el ámbito de la cooperación internacional al desarrollo, sin embargo, permite "realizar un diagnóstico sobre el tipo de actividades, el rol de los actores y las interacciones que se dan entre los actores y eslabones de la cadena para, sobre esa base, generar mejores herramientas de planeación y coordinación o eventuales reposicionamientos en la cadena" (AUCI, 2014, p. 14). En este sentido se puede llevar adelante el concepto de la cadena de valor para la cooperación internacional, tomando en cuenta los objetivos planificados de los actores, las estrategias desarrolladas y el verdadero impacto que tiene la intervención de la cooperación internacional en el desarrollo. El concepto de la cadena de valor no solo revela el valor agregado que generan los actores de la cooperación internacional al desarrollo en cada etapa de la cadena, sino también destaca las debilidades en la misma. El descubrimiento de las debilidades de la cadena de valor de la cooperación internacional permite mejorarlas en el sistema de la cooperación internacional considerando su alta complejidad, baja transparencia y alta lentitud de adaptarse a cambios.

En América Latina el sistema de la cooperación internacional al desarrollo no se encuentra en su mejor momento. Es un sistema que se ha movido en los últimos años entre una cierta comodidad, colado en su zona de confort, y un prejudicio occidental. Este estado institucional ha causado que las debilidades a lo largo de la cadena de valor del sistema de la cooperación internacional al desarrollo se muestran con toda su fuerza. En este sentido se puede empezar con metas mal establecidas que no están realmente alineados a las necesidades verdaderas de los beneficiarios finales de los servicios de la cooperación internacional al desarrollo. Siguiendo con una notable discrepancia entre lo que quiere alcanzar la cooperación internacional en el ámbito de desarrollo y su comprensión del "Desarrollo", buscando a menudo su refugio en la influencia neoliberal. Partiendo desde lo anteriormente mencionado se trata sobre un sistema con una gestión cuestionable con instrumentos y estrategias dudables qué por último ponen en riesgo impactos notables y medibles de una intervención efectiva. Unceta Satrustegui (2013, p. 26) concluye en este sentido que existe una "escasa adaptación de la cooperación, sus objetivos y sus instrumentos, a los retos actuales del desarrollo."

Lo antes mencionado supone desafíos considerables para el sistema de la cooperación internacional al desarrollo. Sin embargo, parte esencial de la búsqueda permanente de autodefinición de la cooperación internacional al desarrollo es el enfoque paradigmático en la eficacia, eficiencia y al final en la efectividad de las acciones y trabajo de sus programas y proyectos al nivel mundial. Para la sociedad internacional una cooperación internacional al desarrollo altamente eficaz y eficiente y al final con una mayor efectividad tiene su razón de ser en el sistema internacional y su existencia en las relaciones internacionales es más justificable y legitima siempre y cuando ella cumple con los indicadores de eficacia, eficiencia y efectividad. Las "3 E" que juzgan sobre el 
desempeño de la intervención de un programa de la cooperación internacional al desarrollo y de esta manera deciden sobre su destino futuro, incluido el riesgo de una aplicación excesiva de estos tres indicadores de desempeño en América Latina.

\section{REFERENCIAS}

Acosta, A. (2010). El buen vivir, una utopía por (re)construir. CIP-Ecosocial - Boletín ECOS, (11), 1-19. https://doi.org/10.14198/OBETS2011.6.1.03

Alesina, A., \& Weder, B. (2002). Do corrupt Governments receive less Foreign Aid? The American Economic Review, 92(4), 1126-1137. https://doi.org/10.1257/00028280260344669

Alonso, J. A., y Ocampo J. A. (2011). Cooperación para el desarrollo en tiempos de crisis. Madrid: Fondo de Cultura Económica de España.

Alonso, J. A. (2001). Nuevas Direcciones en la Política de Ayuda al Desarrollo. Revista de Economía Mundial, (5), 11-45.

Arndt, Ch., Jones, S., \& Tarp, F. (2015). Assessing Foreign Aid's Long - Run Contribution to Growth and Development. World Development, (69), 6-18. https://doi.org/10.1016/j.worlddev.2013.12.016

Agencia Uruguaya de Cooperación Internacional (AUCI) (2014). La Cadena de Valor de la Cooperación Internacional al Desarrollo en Uruguay. Temas de Cooperación (5), 149.

Bauer, P. T. (1972). Dissent on Development. Harvard University Press.

Benito del Pozo, P., y Corcoba, C. (2019). La ayuda oficial al desarrollo y el contexto institucional como factores de crecimiento y progreso: casos comparados de Honduras y Costa Rica. Geopolítica(s). Revista de estudios sobre espacio y poder, 10(1), 87105. https://doi.org/10.5209/GEOP.60942

Cabeza-Pulles, D., y Coral-Guerrero, C. A. (2016). Construction of International Cooperation Strategies: An Inquiry into the learning experience. Iberoamerican $\begin{array}{llll}\text { Journal of Development } & \text { Studies, } & \text { 5(1), }\end{array}$ https://doi.org/10.26754/ojs_ried/ijds.165

Camelo Zamudio, J. A., y Mambuscay, J. D. (2018). Repensar desde el neo institucionalismo la Cooperación Internacional al Desarrollo: Plan Colombia y sus lecciones. Inciso, 19(2), 1-19. https://doi.org/10.18634/incj.20v.1i.858

CEPAL (2010). La Cooperación Internacional en el nuevo Contexto Mundial: Reflexiones desde América Latina y el Caribe. Nota de la Secretaria. Trigésimo Tercer Periodo de Sesiones de la CEPAL. Distr. General, LC / G. 2440 (SES. 3.3 / 11).

Collier, P. (2007). El club de la miseria. Qué falla en los países más pobres del planeta. Debolsillo.

Cunego, A., y Ruiz Sanjuán, S. (2015). Introducción al Sistema de Cooperación Internacional al Desarrollo. Paradigmas, Actores y Perspectivas. Municipalistas por la Solidaridad y el Fortalecimiento Institucional (MUSOL). Agencia Española de Cooperación Internacional (AECID). 
Dollar, D., y Levin, V. (2006). The increasing Selectivity of Foreign Aid, 1984 - 2003. World https://doi.org/10.1016/j.worlddev.2006.06.002

Domínguez, R. (2017). La Alianza para el progreso. Aportes para una teoría crítica de la cooperación. En R. Domínguez, y G. Rodríguez (eds.), Historia de la Cooperación Internacional desde una Perspectiva crítica (pp. 40-57). Red Iberoamericana Académica de Cooperación Internacional (REACI). Debates RIACI. Editorial: Uniautónoma,

Domínguez, R., Said, A., y Valenzuela, E. (2009). Por un nuevo modelo de cooperación pactada al desarrollo en América Latina: For a new model of cooperation agreed to development en Latin America. Revista de Relaciones Internacionales de la UNAM, (105), 63-80.

Duarte Herrera, L. K., González Parias, C. H., y Mesa Bedoya, J. C. (2017). Retos de la Cooperación Internacional para el Desarrollo en Colombia. Estudios Internacionales, 49(188), 111-146. http://dx.doi.org/10.5354/0719-3769.2017.47922

Durán Blanco, E. M. (2008). Eficacias, engaños y autoengaños de la Ayuda Oficial al Desarrollo y las ONGD. La gran mentira de la cooperación internacional. Rebelión. Recuperado de www.rebelion.org/noticias/2008/2/63903]

Easterly, W. (2006). The White Man's Burden: Why the West" s Efforts to Aid the Rest have done so much Ill and so little Good. Pinguin.

Easterly, W., Levine, R., y Roodman, D. (2003). New Data, New Doubts: Revisiting Aid, Policies, and Growth. Working Papers, (26), 1-19.

Encínas, M. A. et al. (2013). La Cooperación Internacional en Tiempos de Crisis. Facultad de Ciencias Políticas y Sociales, Universidad de San Carlos de Guatemala Tricentenaria (USAC). Colección cátedra de coyuntura internacional, III. Cara Parens.

Friedman, M. (1958). Foreign Economic Aid: Means and Objectives. Yale Review, 47(4), 500-516.

Gallicchio, E. (2004). El desarrollo local en América Latina. Estrategia Política basada en la Construcción de Capital Social. Seminario Desarrollo con inclusión y equidad: sus implicaciones desde lo local. Córdoba, Argentina. Recuperado de: https://flacso.edu.ec/cite/media/2016/02/Gallicchio-E_2004_El-desarrollo-local-enAmerica-Latina.-Estrategia-politica-basada-en-la-construccion-de-capital-social.pdf

Gallichio, E. (2004). El desarrollo local: ¿cómo combinar gobernabilidad, desarrollo económico y capital social en el territorio? Cuadernos Del Claeh, 27(89), 55-68. Recuperado de: http://publicaciones.claeh.edu.uy/index.php/cclaeh/article/view/128

Grandas, D., y Prado Lallande, J. P. (2017). La cooperación internacional descentralizada en Colombia. Un análisis institucionalista sobre el departamento de Cundinamarca. Oasis, (25), 159-186. https://doi.org/10.18601/16577558.n25.10

Grandas, D. (2012). Elementos de reflexión para mejorar la práctica de la cooperación oficial descentralizada en los municipios colombianos. Revista de relaciones internacionales, estrategia y seguridad, 7(1), 75-97. https://doi.org/10.18359/ries.92

Gudynas E., y Acosta A. (2011). La renovación de la crítica al desarrollo y el buen vivir como alternativa. Utopía y Praxis Latinoamericana, 16(53), 71-83. 
Gudynas, E. (2011). Buen vivir: Germinando alternativas al desarrollo. América Latina en Movimiento (ALAI), (462), 1-20.

Hayek, F. A. (1953). Substitute for Foreign Aid. Government aid furthers state economic control in Europe and should be replaced by private capital investment backed by limited Government guarantee (pp. 482 - 484). The Freeman. Recuperado de: https://history.fee.org/publications/substitute-for-foreign-aid

Hayter, T. (1971). Aid as imperialism. Penguin Books.

Islam, M. N. (2005). Regime changes, economic policies and the effect of aid on growth. The Journal of Development Studies, 41(8), 1467-1492. https://doi.org/10.1080/00220380500187828

Jiménez-Castillo, M. A. (2018). La encrucijada de la cooperación al desarrollo. Apuestas por una epistemología crítica. Ideas y Valores, 67(167), 223-240. https://doi.org/10.15446/ideasyvalores.v67n167.60232

Juselius, K., y Framroze Møller, N. (2014). The long - run impact of foreign aid in 36 African Countries: Insights from multivariate time series analysis. Oxford Bulletin of Economics and Statistics, 76(2), 153-184. https://doi.org/10.1111/obes.12012

Kasuga, H. (2007). The Millennium Development Goals and Aid Allocation: Which Donors Give High - Quality Aid? Discussion Papers 07050.

Lallande, J. P. (2005). La dimensión ética de la cooperación internacional al desarrollo. Entre la solidaridad y el poder en las relaciones internacionales (pp. 1 -25).

Larrera, C. (2003). La Cooperación Internacional para el desarrollo y sus efectos sociales y económicos en el Ecuador. Observatorio de la Cooperación al Desarrollo en el Ecuador. Comité Ecuménico de Proyectos. Recuperado de: http://www.dhls.hegoa.ehu.eus/uploads/resources/5015/resource_files/cooperacion_i nternacional_para_desarrollo_en_ecuador.pdf

Lensink, R., y White, H. (1999). Assessing Aid: ¿A Manifesto For Aid in the 21 ST Century? Oxford Development Studies, 28(1), 5-18. https://doi.org/10.1080/713688303

Llistar, D. (2009). Anticooperación. Interferencias Norte - Sur. Los problemas del Sur Global no se resuelvan con más ayuda internacional. Icaria - Antrazyt Relaciones Norte - Sur.

Lo Brutto, G. (2017). A Propósito de la Cooperación Internacional y del Desarrollo: Una Visión más realista. En R. Domínguez, y G. Rodríguez Albor (eds.), Historia de la Cooperación Internacional desde una Perspectiva crítica (pp. 55-73). Red Iberoamericana Académica de Cooperación Internacional (REACI). Debates RIACI. Uniautónoma.

Lucas, J. M., y Franco, D. C. (2017). La Cooperación Oficial europea y española ante el nuevo escenario del sistema internacional de cooperación al desarrollo. Acciones $e$ Investigaciones Sociales, (37), 7-25.

Lucatello, S. (2017). La Solidaridad Tóxica en América Latina: ¿Cooperación al Desarrollo o Industria de la Ayuda? Una Trama en tres Actos. En R. Domínguez, y G. Rodríguez (eds.), Historia de la Cooperación Internacional desde una Perspectiva crítica (pp. 165-194). Red Iberoamericana Académica de Cooperación Internacional (REACI). Debates RIACI. Uniautónoma. 
Maren, M. (2007). The Road to Hell: The Ravaging Effects of Foreign Aid and International Charity. Kindle Edition.

Mekasha, T., \& Tarp, F. (2013). Aid and Growth What Meta-Analysis Reveals. Journal of Development Studies, $49(4), \quad 564-583$. https://doi.org/10.1080/00220388.2012.709621

Mende, T. (1973). From aid to re - colonization: lessons of a failure. Pantheon Books.

Millán, N., Gutiérrez, J., y Sabalza, M. (2015). Tema Central, Coherencia de Políticas para el Desarrollo: más allá de la AOD. Centro de Documentación HEGOA. Boletín de Recursos de Información, (44), 1-10.

Monje, J. A. (2018). Antropología del desarrollo y factores críticos para el éxito de los proyectos de cooperación internacional. El caso de las ONGD en América Latina. Revista de Antropología Iberoamericana, 13(1), 93-117. https://doi.org/10.1080/713688303

Moore Lappe, F., Collins, J., y Kinley, D. (1981). Aid as obstacle. Twenty questions about our foreign aid and the hungry. Institute for Food and Development Policy San Francisco. 6(3), 1-192.

Morgenthau, H. (2015). Una teoría política sobre la ayuda exterior. Relaciones Internacionales, (28), 147-162.

Moyo, D. (2011). Cuando la ayuda es el problema. Hay otro camino para África. Gota a Gota.

Muñiz de Urquiza, M. (2002). La Cooperación para el Desarrollo con América Latina en el Marco de la Política Exterior de la España Democrática (Tesis Doctoral). Departamento de Estudios Internacionales. Facultad de Ciencias Políticas y Sociológica. Universidad Complutense de Madrid (España).

Muñoz Ocaña, Y. (2013). Análisis de la Ayuda Oficial al Desarrollo para la Cubertura de las Necesidades Sociales Básicas (Tesis Doctoral). Departamento de Gestión Empresarial y Métodos Cuantitativos. Centro Ciencias Económicas y Empresariales (ETEA), Centro adscrito a la Universidad de Córdoba. Universidad de Córdoba (España).

Narros Lluch, A. (2015). La comedia del Desarrollo Internacional en la Isla de Camiguin (Filipinas): Historias etnográficas sobre impacto y sostenibilidad (Tesis Doctoral). Departamento de Antropología Social y Cultural. Facultad de Filosofía. Universidad Nacional de Educación a Distancia (UNED).

Nozick, R. (1974). Anarchy, State and Utopia. Blackwell Publishers.

Oetzel, R. (2009). Cooperación Internacional: recuperar la política. Actuar en mundos plurales, (5), 10-13. Recuperado de: https://repositorio.flacsoandes.edu.ec/bitstream/10469/751/1/BFLACSO-AMP5-04Oetzel.pdf

Olivié, I., y García, E. (2010). ¿Dónde debe ir la Ayuda? Propuesta de un Índice de Desarrollo para la Asignación Internacional de la Ayuda. Revista de Economía Mundial, (24), 131-162.

Oroval, C. C. (2008). La Cooperación al Desarrollo: ¿Un concepto agotado o mal usado?, $1-3$. 
Pearson, L. B. (1970). The Pearson Report. A new Strategy for Global Development. A Window open on the world. Courier. The UNESCO, (23), 1-40.

Posadas Mijangos, K. T. (2013). Nuevas modalidades de cooperación que ejecutan los principales donantes en Guatemala. En M. A. Encías et al., La Cooperación Internacional en Tiempos de Crisis (pp. 99-130). Facultad de Ciencias Políticas y Sociales, Universidad Rafael Landívar. Cara Parens.

Restrepo, M. (2012). La Cooperación internacional al desarrollo como herramienta de protección y promoción de los derechos humanos: el caso latinoamericano. Revista Facultad de Derecho y Ciencias Políticas, 42(116), 271-295.

Riddel, R. C. (2007). Does Foreign Aid Really Work? Oxford University Press.

Ripoll, A., y Ghotme, R. (2015). La cooperación internacional: herramienta de desarrollo o de atraso. Revista Latinoamericana de Bioética, 15(1), 54-63.

Sábato, J. A., Caputo, D., y Sábato, J. F. (1981). Cooperación para el desarrollo: Algunas reflexiones y propuestas. Estudios internacionales, 17-47.

Sachs, J. (2004). The End of Poverty: Economic Possibilities for Our Time. Penguin Press.

Sogge, D. (2009). Sistema de ayuda extranjera: ¿Régimen o Vehículo Hegemónico?, Relaciones Internacionales, (12), 11-31.

Sow, C. B. (2016). Cooperación al Desarrollo y Derechos Humanos (en el Ámbito de las Relaciones Internacionales). Tesis Doctoral. Universidad de Jaén. Facultad de Ciencias Sociales y Jurídicas, Departamento de Derecho Público y Derecho Privado Especial.

Temple, J. (2010). Aid and Conditionality. In D. Rodrik and M. Rosenzweig (eds), Handbook of Development Economics, 5(1), 4415-4523.

Thiele, R., Nunnenkamp, P., \& Dreher, A. (2007). Do Donors Target Aid in Line with the Millennium Development Goals? A Sector Perspective of Aid Allocation. Review of World Economics, 143(04), 596-630. https://doi.org/10.1007/s10290-007-0124-X

Torres, R. M. (2000). Cooperación Internacional en educación en América Latina: ¿Parte de la solución o parte del problema? La "cooperación internacional” en la mira (pp. 1-14). Pronunciamiento Latinoamericano sobre Educación para Todos. Recuperado de:

https://pdfs.semanticscholar.org/b1c4/00050a5df6cb08a1324fccb8b5d8fe5a45b2.pdf

Unceta, K. (2013). Cooperación para el desarrollo: anatomía de una crisis. Íconos. Revista de Ciencias Sociales, (47), 15-29. https://doi.org/10.17141/iconos.47.2013.820

Unceta, K., y Guitiérrez, J. (2012). Identidad y legitimitad de la cooperación al desarrollo: el debate sobre la relación de la AOD con la pobreza y la desigualdad internacional. Estudios de Economía Aplicada, 30(3), 773-800.

Unceta, K., y Yoldi, P. (2000). La Cooperación al Desarrollo: surgimiento y evolución Histórica. Cooperación Pública Vasca. Gobierno Vasco.

Vassalli, G., y Rodríguez Albor, G. (2017). La Cooperación Internacional en el Asimétrico Sistema Global Neoliberal: Un Análisis histórico-rítico desde el Sur. En R. Domínguez, y G. Rodríguez Albor (eds.), Historia de la Cooperación Internacional desde una Perspectiva crítica (pp. 77-102). Red Iberoamericana Académica de Cooperación Internacional (REACI). Debates RIACI. Uniautónoma. 
Zamora Aviles, E. A. (2013). Elementos críticos sobre cooperación internacional en el Magdalena Medio Colombiano. Íconos Revista de Ciencias Sociales, (47), 47-67. 